\title{
$\alpha$ CGRP-Induced Changes in Cerebral and Systemic Circulation; A TCD Study
}

\author{
Darja Visočnik*, Bojana Žvan, Marjan Zaletel and Matija Zupan \\ Department of Neurology, University Medical Center Ljubljana, Ljubljana, Slovenia
}

It is known that perivascular application of CGRP induces cerebral vasodilatation. However, it is unclear whether intravenous alfa CGRP ( $\alpha$ CGRP) induces changes in cerebral and systemic hemodynamics. Therefore, we studied the influence of an $\alpha$ CGRP intravenous infusion at a rate of $1.5 \mathrm{mcg} / \mathrm{min}$ in $20 \mathrm{~min}$ on mean arterial velocity in the middle cerebral artery (vm MCA) and in the posterior cerebral artery (vm PCA) in twenty healthy subjects using transcranial Doppler (TCD). We found out that $\alpha \mathrm{CGRP}$ decreased vm MCA $(p<0.001)$, vm PCA $(p<0.001)$, mean arterial pressure (MAP) $(p<0.001)$ and end-tidal $\mathrm{CO}_{2}\left(\mathrm{Et}-\mathrm{CO}_{2}\right)(p=0.030)$. The heart rate $(\mathrm{HR})$ increased during $\alpha$ CGRP infusion $(p<0.001)$. In addition, we found a positive relationship between Et-CO $\mathrm{CO}_{2}$ and vm MCA $(p=0.001)$ as well as vm PCA $(p=0.043)$. In our view, $\alpha$ CGRP induces changes in cerebral and systemic circulation in healthy volunteers. It might cause vasodilatation of MCA and PCA and a compensatory decrease of Et- $\mathrm{CO}_{2}$ to $\alpha \mathrm{CGRP}$ related hemodynamic changes.

Massimiliano Valeriani, Bambino Gesù Children Hospital (IRCCS), Italy

Keywords: calcitonin gene-related peptide, migraine, cerebral arteries, transcranial doppler sonography, systemic hemodynamic

Reviewed by:

Simona Sacco,

University of L'Aquila, Italy

Claudia Altamura,

Campus Bio-Medico University, Italy

*Correspondence:

Darja Visočnik

darja.visocnik@kclj.si

Specialty section:

This article was submitted to Headache Medicine and Facial Pain,

a section of the journal

Frontiers in Neurology

Received: 30 June 2020 Accepted: 03 September 2020

Published: 06 November 2020

Citation:

Visočnik D, Žvan B, Zaletel M and Zupan M (2020) aCGRP-Induced

Changes in Cerebral and Systemic

Circulation; A TCD Study.

Front. Neurol. 11:578103.

doi: 10.3389/fneur.2020.578103

\section{INTRODUCTION}

Calcitonin gene-related peptide (CGRP) has been proven to play an important role in migraine and it has therefore become a target molecule in headache migraine research and treatment $(1-5)$. There is growing evidence that alfa CGRP $(\alpha$ CGRP) importantly affects clinical and physiologic states.

Recently, it has been shown that patients with an excellent response to erenumab are highly susceptible to $\alpha$ CGRP provocation (6). Therefore, an $\alpha$ CGRP response should be regarded as an important predictive factor for selecting patients who are responders to anti-CGRP preventive treatment. In addition, current evidence suggests that CGRP is a potent dilatator of cerebral arteries (7-9). However, it is still unclear whether vasodilatation caused by $\alpha$ CGRP through intravascular interaction with an endogenous CGRP system, utilizes vascular endothelium.

Until now there have only been a few studies conducted on the effects of intravenous $\alpha \mathrm{CGRP}$ on middle cerebral artery (MCA) dilatation and none on the response of the posterior cerebral artery (PCA) on CGRP. It has been shown that endothelium of anterior and posterior cerebral circulation could differ in function (10). Regarding cerebral effects of aCGRP, some studies demonstrated vasodilatation of MCA in response to intravenous $\alpha$ CGRP $(7,8)$, while others did not (9). In addition, the effect of $\alpha$ CGRP on systemic hemodynamics has been proven in animal and human studies. Earlier studies showed that $\alpha$ CGRP is a potent microvascular vasodilator but may not play an important role in the physiological control of systemic blood pressure (11). On the other hand, some studies have shown a significant decrease of systemic arterial pressure after receiving intravenous $\alpha$ CGRP (7). Thus, the cerebral and systemic effects of intravascular 
aCGRP are not completely understood. In addition, in the previous transcranial Doppler (TCD) studies in healthy volunteers for $\alpha$ CGRP effects on MCA mean velocity, a change in the $\mathrm{CO}_{2}$ concentration in exhaled air $\left(\mathrm{Et}-\mathrm{CO}_{2}\right)$ during intravenous $\alpha$ CGRP infusion was observed. The Et- $\mathrm{CO}_{2}$ significantly decreased during aCGRP application, but not when volunteers were treated with a placebo $(7,8)$. However, this phenomenon was not discussed and systematically studied. The polymodal monitoring of cerebral and systemic hemodynamic parameters is necessary to evaluate the hemodynamic effects of $\alpha$ CGRP stimulation. The aim of our study was to assess the influence of intravenous aCGRP on cerebral and systemic hemodynamic parameters using the TCD method. We hypothesize that intravenous aCGRP may have a significant vascular effect on both MCA and PCA and on systemic circulation.

\section{MATERIALS AND METHODS}

Twenty healthy subjects participated in our study (nine females aged $37.0 \pm 2.8$ years, 11 males aged $41.8 \pm 7.6$ years, $p=0.66$ ). The inclusion criteria were age more than 18 years, normal somatic and neurological status, normal laboratory tests (complete blood count, serum potassium and sodium), and no atherosclerotic process of the carotid and vertebral arteries excluded by color-coded duplex sonography. The exclusion criteria were migraine and other primary headache disorders, previous cerebrovascular, endocrine, renal or liver diseases, uncontrolled hypertension, daily intake of medication except for contraceptives, pregnancy and breast-feeding.

The participants were free of tobacco, coffee, tea or any other food or beverages containing caffeine for at least $12 \mathrm{~h}$ before the start of the measurements. The color-coded duplex sonography of the carotid and vertebral arteries excluded an early atherosclerotic process in participants.

All participants were given written explanations about the experimental procedure and were informed that they were free to withdraw from the study at any time. They all gave written informed consent to participate in the study. The National Medical Ethics Committee of the Republic of Slovenia approved the study.

The experiments took place at 9:00 am in a quiet and dark room with constant temperature. During the experiment, the participants were resting in the supine position. Transcranial Doppler (TCD) sonography with $2 \mathrm{MHz}$ ultrasound probes was applied to measure the mean flow velocity in MCA (vm MCA) through the left and mean flow velocity in PCA (vm PCA) through the right temporal acoustic window. The signals of the arteries were defined according to the direction of the blood flow, the typical depth of the signal and the response to compression. A mechanical probe holder was used to ensure a constant probe position. During the entire experiment the mean blood pressure (MAP) and heart rate (HR) were continuously measured using non-invasive plethysmography (Colin 7000, Komaki, Japan). The Et- $\mathrm{CO}_{2}$ was measured by an infrared capnograph (Capnograph, Model 9004, Smith medical, USA) using the standard protocol.
The capnograph was connected to a breathing mask and to the computer. Et- $\mathrm{CO}_{2}$ signals were recorded on the same time scale as other variables. This enabled us to compare the signals and perform correlations between them.

The experiment consisted of a $10 \mathrm{~min}$ baseline period, a 20 min period during which an intravenous infusion of $\alpha$ CGRP $1.5 \mathrm{mcg} / \mathrm{min}$ (Calbiochem, Merck4Biosciences, Darmstadt, Germany) was given and a $10 \mathrm{~min}$ period after the end of the $\alpha$ CGRP infusion. TCD Multi-Dop X4 software (DWL, Sipplingen, Germany) was used to define average values of all parameters (vm MCA and vm PCA, MAP, HR and Et- $\mathrm{CO}_{2}$ ) during $5 \mathrm{~min}$ intervals. The first interval - point 0 was during the baseline period before starting $\alpha$ CGRP infusion (5-10 min of the experiment). The second interval - point 1 was 5-10 min after the start of $\alpha$ CGRP infusion (15-20 min of the experiment). The third interval - point 2 was 15-20 min after the start of $\alpha$ CGRP infusion (25-30 min of the experiment). The last interval - point 3 was 5-10 min after the end $\alpha$ CGRP infusion (35-40 min of the experiment). The mean vm MCA and vm PCA was calculated for each $5 \mathrm{~min}$ interval using the following equation $\operatorname{vm} \int=$ $\mathrm{vdt} /(\mathrm{t} 0-\mathrm{t} 5)$. The mean values of other variables (MAP, HR, Et$\mathrm{CO}_{2}$ ) were also calculated for the same time intervals as the flow velocity using TCD software.

Sample size was driven from previous studies. For statistical analysis, SPSS version 21 was used. ANOVA for repeated measures was used to test differences among the successive points and a paired $t$-test was used to test the significance of differences between two points. Linear regression was used to test the correlations between the variables. All variables had value of the Shapiro-Wilk test $>0.05$.

\section{RESULTS}

First, we analyzed the vm MCA, vm PCA, MAP, HR, and Et$\mathrm{CO}_{2}$ differences between the measurement points using the paired $t$-test. The statistical charts of the signals are presented in Figure 1. The probabilities ( $p$-values) of differences between chosen points are shown in Table $\mathbf{1 .}$

The vm MCA showed a significant decrease in all points except between points $1-3$ and 2-3 because of a slight increase of the signal at the recovery phase, after the end of the $\alpha$ CGRP infusion. The decrease of vm MCA was steady between points 1 and 2. The minimum value of vm MCA was measured at the point 2. The vm PCA changed in a similar way during the $\alpha$ CGRP infusion, except for vm PCA decreasing between points 1 and 3, which appeared to be significant in vm PCA but not in vm MCA (Figures 1A,B).

The MAP decreased during the aCGRP infusion and increased after it. The decrease was significant between measuring points 0 and 2, i.e., at the time of the CGRP infusion. The trends between points $0-1$ and 1-2 were not significant. However, we observed a significant response of MAP between the points $1-3$ and $2-3$. There was no difference between points 0 and 3, which showed similar values for the MAP signal before and after the $10 \mathrm{~min}$ after the end of the $\alpha$ CGRP infusion (Figure 1C). HR significantly increased during the $\alpha$ CGRP infusion between 
A

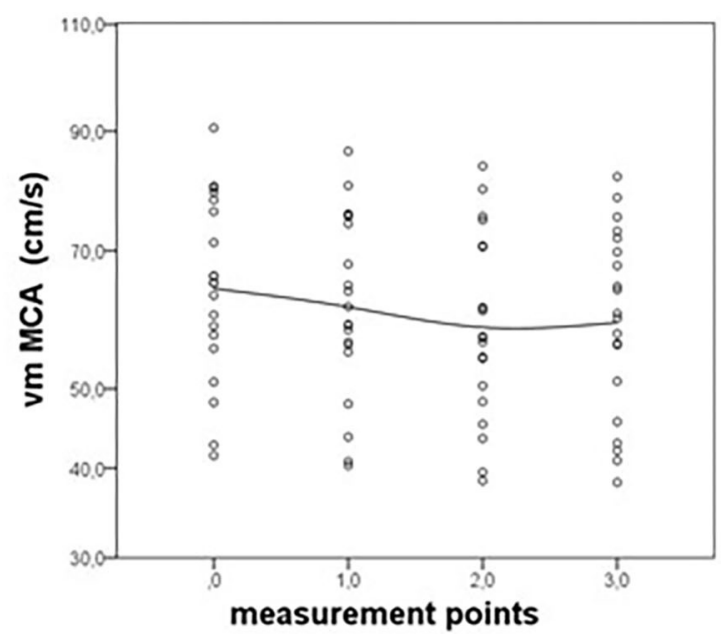

C

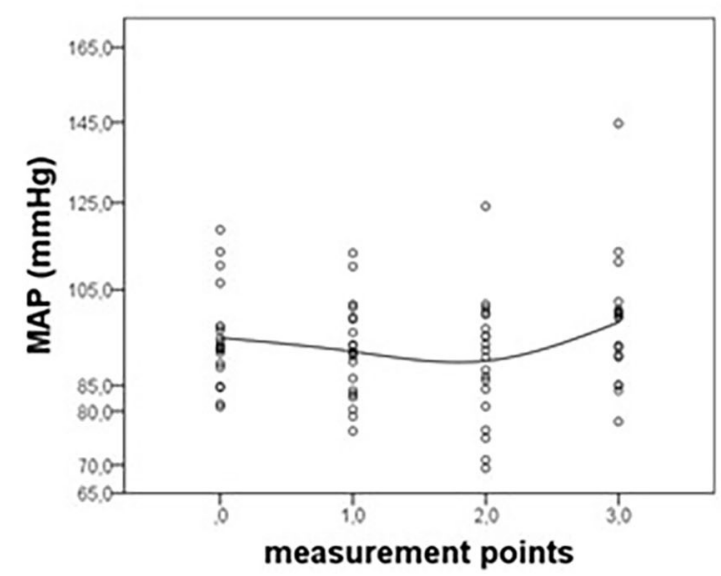

B

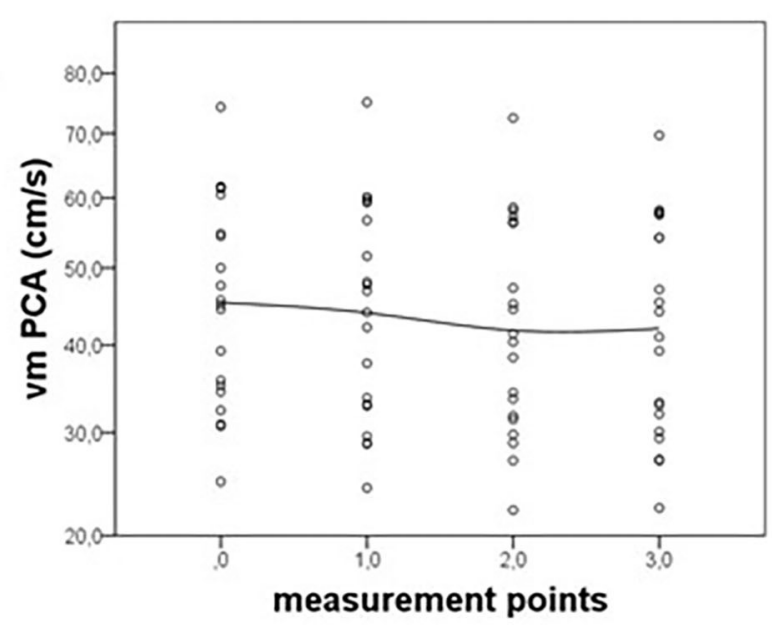

D

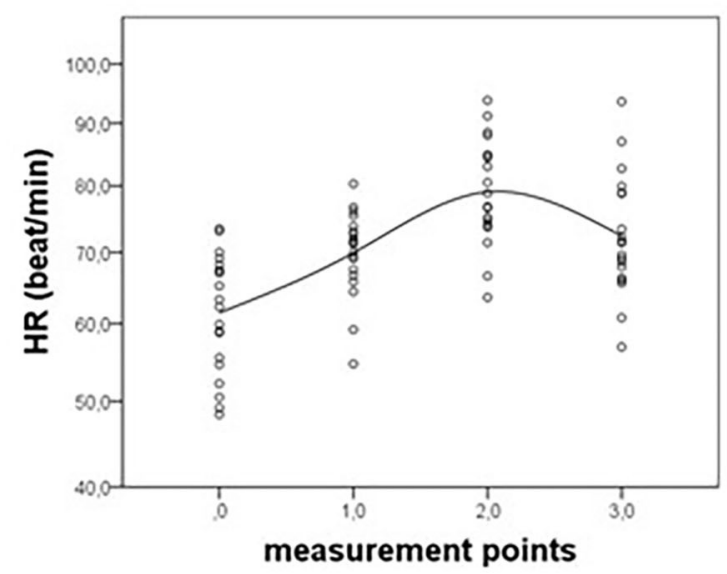

E

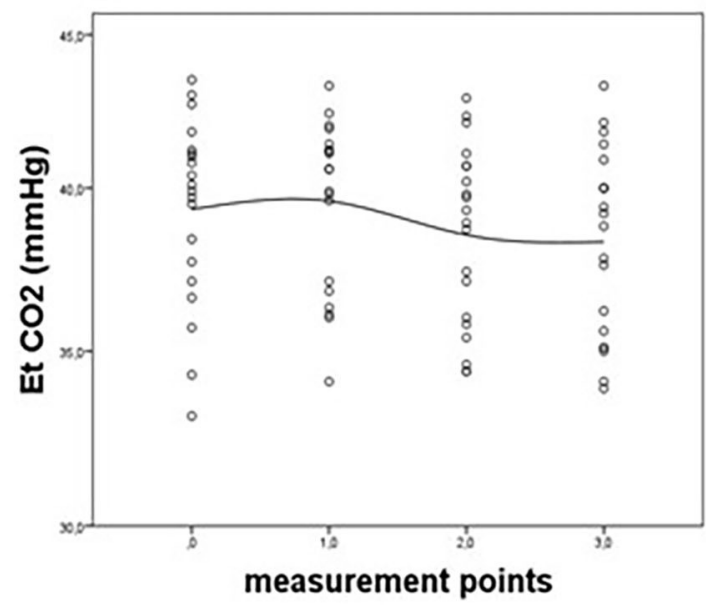

FIGURE 1 | Statistical charts of (A) mean arterial velocity in middle cerebral artery (vm MCA), (B) mean arterial velocity in posterior cerebral artery (vm PCA), (C) mean arterial pressure (MAP), (D) heart rate (HR), and (E) end tidal carbon dioxide (Et- $\left.\mathrm{CO}_{2}\right)$ before (point 0), during (points 1 and 2), and after CGRP infusion (point 3). 
TABLE 1 | The probability of differences ( $p$-values) between measuring points using paired-t-test for vm MCA, vm PCA, MAP, $\mathrm{HR}$, and $\mathrm{Et}-\mathrm{CO}_{2}$.

\begin{tabular}{lccccc}
\hline Pair points & $\begin{array}{c}\text { vm MCA } \\
\text { ( } \boldsymbol{p} \text {-value) }\end{array}$ & $\begin{array}{c}\text { vm PCA } \\
\text { (p-value) }\end{array}$ & $\begin{array}{c}\text { MAP } \\
\text { (p-value) }\end{array}$ & $\begin{array}{c}\text { HR } \\
\text { ( } \text {-value) }\end{array}$ & $\begin{array}{c}\text { Et-CO } \\
\text { (p-value) }\end{array}$ \\
\hline $0-1$ & $<0.001$ & 0.003 & 0.062 & $<0.001$ & 0.376 \\
$0-2$ & $<0.001$ & $<0.001$ & 0.027 & $<0.001$ & 0.023 \\
$0-3$ & $<0.001$ & 0.002 & 0.119 & $<0.001$ & 0.066 \\
$1-2$ & $<0.001$ & 0.003 & 0.105 & $<0.001$ & 0.004 \\
$1-3$ & 0.062 & 0.020 & 0.001 & 0.075 & 0.016 \\
$2-3$ & 0.401 & 0.710 & $<0.001$ & $<0.001$ & 0.463 \\
\hline
\end{tabular}

vm MCA, mean arterial velocity in middle cerebral artery; vm PCA, mean arterial velocity in posterior cerebral artery; MAP, mean arterial pressure; $\mathrm{HR}$, heart rate; $\mathrm{Et}-\mathrm{CO}_{2}$, end tidal carbon dioxide.

points $0-1$ and $1-2$. After the end of the $\alpha$ CGRP infusion, the HR significantly decreased, which is represented by a significant difference between points 2 and 3. The final level of HR was similar to that in point 1 because we did not detect a significant difference between points 1 and 3 (Figure 1D).

The third part of our analysis was changes in Et- $\mathrm{CO}_{2}$ associated with the $\alpha$ CGRP infusion. The signal showed a remarkable and significant decrease during the $\alpha$ CGRP infusion between points $0-2$ and 1-2. After the end of the infusion, there was no significant change of Et- $\mathrm{CO}_{2}$, which is between points 2 and 3 (Figure 1E).

Using linear regression, we analyzed correlations between vm MCA, vm PCA, MAP, HR and Et- $\mathrm{CO}_{2}$. We found significant positive correlations between vm MCA, vm PCA and Et- $\mathrm{CO}_{2}$. The correlation between vm MCA and vm PCA was significant. We did not find any significant correlations between mean velocity in both cerebral arteries and MAP and HR (Table 2). We found a significant negative correlation between Et- $\mathrm{CO}_{2}$ and $\mathrm{HR}$ as well as MAP. But we did not establish a relationship between MAP and HR. The results are presented in Table 2.

\section{DISCUSSION}

The main finding of our study was a significant decrease of vm MCA and vm PCA during the $\alpha$ CGRP infusion at a rate of $1.5 \mathrm{mcg} / \mathrm{min}$ for $20 \mathrm{~min}$ (Figures 1A,B). The decrease of mean velocity in both arteries was steady through the entire $\alpha$ CGRP infusion with the lowest values just before the end of the infusion. After the end of the infusion, the velocities in both territories tended to increase, although insignificantly.

The arterial velocity reflects the relationship between blood flow and the cross-sectional area of the insonated artery. In the case where this relationship is constant and therefore the arterial velocity is constant, the dilatation of the artery due to CGRP effect should be followed by a proportional increase in cerebral blood flow (CBF). However, we found that during the $\alpha$ CGRP infusion, vm in MCA and PCA decreased. In addition, we found a highly positive correlation between vm MCA and vm PCA. Accordingly, the relationship between cerebral blood flow and arterial cross-sectional area also decreased. This could be due to proximal arterial vasodilatation or a decrease in $\mathrm{CBF}$. In healthy volunteers, $\alpha$ CGRP increased global and regional CBF (8), but in migraine patients, there was no $\alpha$ CGRP effect on regional $\mathrm{CBF}$. In our study, CBF was not measured because we wanted to evaluate an uncomplicated method for routine usage. Regardless of changes in CBF, we assume that the decrease in flow velocity induced by intravenous $\alpha$ CGRP might be evidence of MCA and PCA dilatation.

Our assumption is supported by results of extensive in vitro and in vivo research in animal and human studies that provide evidence that CGRP is a potent vasodilator and has greater potency compared to prostaglandin, acetylcholine and substance P (11). CGRP probably causes dilatation of cerebral arteries but has no effect on cerebral veins (12). Our results support the thesis that $\alpha$ CGRP induces dilatation of proximal cerebral arteries such as MCA and PCA. Indeed, previous studies showed that $\alpha$ CGRP is associated with MCA vasodilatation $(7,8)$. Lassen et al. found that $\alpha$ CGRP infusion dilates MCA in patients with migraines. The vm MCA significantly decreased during the $\alpha$ CGRP infusion at a rate of $2 \mathrm{mcg} / \mathrm{min}$ for $20 \mathrm{~min}$ in migraine patients compared to healthy subjects. The lowest value of vm MCA was measured 15 min after the start of the infusion, which was the last measurement during the $\alpha$ CGRP infusion. The regional $\mathrm{CBF}$ ( $\mathrm{rCBF}$ ) was measured using ${ }^{133} \mathrm{Xe}$ SPECT at the baseline condition and after $15 \mathrm{~min}$ of the $\alpha$ CGRP infusion and it did not change during the $\alpha$ CGRP infusion. Petersen et al. in a study comparing efficacy of the CGRP receptor antagonist, BIBN4096BS, and a placebo in healthy volunteers, also found MCA dilatation caused by $\alpha$ CGRP infusion. In fact, their study was not set up to rule out $\alpha$ CGRP effects on MCA; however, the global CBF, rCBF and vm MCA was measured during and after the $\alpha C G R P$ infusion at a rate of $1.5 \mathrm{mcg} / \mathrm{min}$ for $20 \mathrm{~min}$. The vm MCA was constant during the $\alpha$ CGRP infusion, but the global $\mathrm{CBF}$ and the $\mathrm{rCBF}$ increased after $\alpha \mathrm{CGRP}$ administration. They calculated the relative percentage change of diameter of MCA and estimated that $\alpha$ CGRP increased the MCA diameter (8).

On the other hand, the study by Asghar did not show any change in the MCA circumference during an aCGRP infusion (9). In this study magnetic resonance angiography (MRA) was used to measure the changes of MCA circumference. The measurements were done before an $\alpha$ CGRP infusion and $10 \mathrm{~min}$ after the end of it. In our study, we found that after the end of the aCGRP infusion the velocities in MCA and PCA tended to increase, which could be evidence of arterial diameter normalization. Lassen also found no difference in vm MCA between the CGRP and the placebo group at the last measurement of vm MCA, 75 min after the start of the $\alpha$ CGRP infusion (7). The discrepancy between the Asghar study and our data could be due to the timing of MRA measurements. We suspect that the delay in MRA after the end of the CGRP stimulation attributed to their conclusions. Furthermore, the accuracy of MRA in the assessment of small but significant changes in MCA circumference is still not clarified (13).

At present, the mechanism of the $\alpha$ CGRP vasodilatation effect is only partly understood. Earlier studies in animals and in vitro in humans revealed that perivascular application of $\alpha$ CGRP induces dilatation of cerebral arteries (14). However, 
TABLE 2 | Linear correlations between vm MCA, vm PCA, MAP, HR, and Et- $\mathrm{CO}_{2}$.

\begin{tabular}{|c|c|c|c|c|c|}
\hline & vm MCA & vm PCA & MAP & HR & $\mathrm{Et}-\mathrm{CO}_{2}$ \\
\hline \multirow[t]{3}{*}{ vm MCA } & & Positive & Positive & Negative & Positive \\
\hline & & $p<0.001$ & $p=0.744$ & $p=0.338$ & $p=0.001$ \\
\hline & & $r=0.645$ & $r=0.037$ & $r=0.109$ & $r=0.372$ \\
\hline \multirow[t]{3}{*}{ vm PCA } & Positive & & Positive & Positive & Positive \\
\hline & $p<0.001$ & & $p=0.081$ & $p=0.281$ & $p=0.043$ \\
\hline & $r=0.645$ & & $r=0.197$ & $r=0.123$ & $r=0.227$ \\
\hline \multirow[t]{3}{*}{ MAP } & Positive & Positive & & Positive & Negative \\
\hline & $p=0.744$ & $p=0.081$ & & $p=0.111$ & $p=0.012$ \\
\hline & $r=0.037$ & $r=0.197$ & & $r=0.183$ & $r=0.280$ \\
\hline \multirow[t]{3}{*}{$\mathrm{HR}$} & Negative & Positive & Positive & & Negative \\
\hline & $p=0.338$ & $p=0.281$ & $p=0.111$ & & $p=0.007$ \\
\hline & $r=0.109$ & $r=0.123$ & $r=0.183$ & & $r=0.300$ \\
\hline \multirow[t]{3}{*}{$\mathrm{Et}-\mathrm{CO}_{2}$} & Positive & Positive & Negative & Negative & \\
\hline & $p=0.001$ & $p=0.043$ & $p=0.012$ & $p=0.007$ & \\
\hline & $r=0.372$ & $r=0.227$ & $r=0.280$ & $r=0.300$ & \\
\hline
\end{tabular}

The words positive or negative represent direction of correlations.

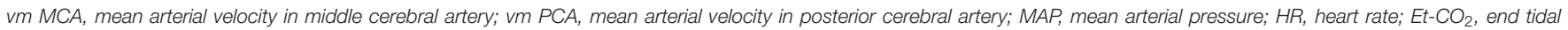
carbon dioxide.

aCGRP given luminally failed to induce dilation of rat MCA (15). These findings are in accordance with the confirmed localization of CGRP receptors in arterial wall and support the opinion that CGRP-receptor components are present only in the cerebrovascular smooth muscle cells in arterial walls rather than in the cerebral endothelium. Furthermore, CGRP containing trigeminal nerves have been shown to surround the major cerebral and cortex pial arteries and that their stimulation releases CGRP, which in turn causes direct vasodilation via the receptors located on the vascular smooth muscle cells. On the other hand, it was confirmed that intravenous $\alpha$ CGRP causes dilatation of MCA and the middle meningeal artery as well (7-9). Furthermore, circulating CGRP receptor antagonists and antibodies against CGRP and the CGRP receptor, all of which are effective in migraine treatment, do not seem to be able to cross the blood-brain barrier to access targets in the cerebral vasculature (11). The results of our study speak in favor of an intravascular mechanism of $\alpha$ CGRP or effect at the regions outside the bloodbrain barrier such as trigeminal ganglion. The cerebral arteries of migraineurs show enhanced response to exteroceptive stimuli probably mediated by CGRP (16). Therefore, we would expect more intensive response of arterial velocity and Et- $\mathrm{CO}_{2}$ to CGRP in a patient with migraine.

The next important finding of our study is that intravenous infusion of $\alpha$ CGRP at a rate of $1.5 \mathrm{mcg} / \mathrm{min}$ for $20 \mathrm{~min}$ significantly decreases MAP. The maximal decrease of MAP was at the end of the infusion. After that, a marked increase in MAP was observed. Changes in HR were also significant and in the opposite direction to the changes of MAP. The linear relationship between the variables was inconclusive. From this, we conclude that intravenous $\alpha$ CGRP decreases systemic blood pressure and causes an increase in HR, which is most probably due to cardiac output compensation of the decrease in MAP.
Our results support the systemic effects of $\alpha$ CGRP in humans. In animal and human studies, intravenous administration of CGRP was associated with a decrease in systemic blood pressure and positive inotropic and chronotropic heart responses $(11,17)$. Furthermore, in our study no associations between MAP and vm MCA, as well as between MAP and vm PCA were found, which indicates uncoupling between cerebral flow and systemic arterial pressure and therefore normal regulation of $\mathrm{CBF}$ during and after $\alpha$ CGRP stimulation.

The interesting finding of our study was also that the Et$\mathrm{CO}_{2}$ during the $\alpha \mathrm{CGRP}$ infusion decreased significantly and persistently even after the end of the $\alpha$ CGRP infusion. The Et- $\mathrm{CO}_{2}$ decrease was reported in migraine patients during $\alpha$ CGRP infusion but not when treated with a placebo. The peak decrease in Et- $\mathrm{CO}_{2}$ occurred $5 \mathrm{~min}$ after the end of the $\alpha$ CGRP infusion (7). It seems that an Et- $\mathrm{CO}_{2}$ decrease does not occur incidentally. We also found positive and significant relationships between Et- $\mathrm{CO}_{2}$ and vm MCA as well as Et$\mathrm{CO}_{2}$ and vm PCA. According to current knowledge, vm in MCA and PCA largely depends on cerebral arterioles status. Thus, $\alpha$ CGRP provokes important vasodilation at the arterioles' levels with the consequent putative increases in vm MCA and vm PCA. On the other hand, vasodilation/constriction of arterioles by $\mathrm{CO}_{2}$ concentration strongly determinates vm MCA and vm PCA. This is a well-known phenomenon named vasomotor reactivity to hyper/hypocapnia and it is one of the main control mechanisms to ensure constant cerebral perfusion $(18,19)$. Thus, depending on the stimulus, the vasodilatation could be largely distal, for example during $\mathrm{pCO}_{2}$ changes or largely proximal, for example the response to nitro-glycerine, or it could be distal and proximal (20). Therefore, we assume that a decrease in Et- $\mathrm{CO}_{2}$ during $\alpha \mathrm{CGRP}$ stimulation tends to decrease $\mathrm{CBF}$ and consequently arterial 
velocities in MCA and PCA. The Et- $\mathrm{CO}_{2}$ decrease during the $\alpha$ CGRP stimulation could represent the compensation of an aCGRP-induced increase of cerebral blood flow in order to preserve intracranial pressure in the normal range. We could infer that Et- $\mathrm{CO}_{2}$ acts predominantly on distal resistant cerebral vessels, whereas aCGRP induces vasodilatation in proximal and distal parts of the cerebral arterial tree with a net decrease in arterial velocities in MCA and PCA. In other words, the relationship between blood flow and the cross-sectional area of the insonated artery decreased due to the vasodilatory properties of $\alpha \mathrm{CGRP}$ on MCA and PCA. Moreover, we found significant negative relationships between Et- $\mathrm{CO}_{2}$ and MAP as well as Et- $\mathrm{CO}_{2}$ and $\mathrm{HR}$. This could be explained by the common effect of $\alpha \mathrm{CGRP}$ on Et- $\mathrm{CO}_{2}$, MAP and $\mathrm{HR}$ and not by causal relationships between the variables. Both Et- $\mathrm{CO}_{2}$ and $\mathrm{HR}$ seem to have a compensatory role in cerebral blood flow and systemic arterial pressure regulation, which have to be homeostatically preserved during an $\alpha$ CGRP infusion.

It is known that CGRP is derived predominantly from perivascular sensory neurons and the CGRP receptor complex was shown to be located on the smooth muscle cells of the cerebral vasculature. In addition, studies have shown that perivascular application of CGRP induced vasodilatation even after endothelial removal (21). The dilatory effect of CGRP on feline cerebral arteries is clearly endothelium independent, and the same is true for human cerebral, meningeal and temporal arteries (15). Furthermore, abluminal but not luminal application of $\alpha$ CGRP caused concentration-dependent vasodilatation of rat MCA. The results of these studies suggest the endothelialindependent action of CGRP. On the other hand, some studies confirm the endothelium-mediated vasodilatation of CGRP. In the periphery, CGRP and its receptors are present in endothelial cells and CGRP promotes the proliferation of endothelial cells (22). Human studies in vivo demonstrated the vasodilatation of cerebral arteries, and the medial meningeal and superficial temporal artery after intravenous infusion of $\alpha$ CGRP. Our study supports the intravascular effects of $\alpha$ CGRP probably via intravascular endothelium. In our study, we showed the effects of CGRP on cerebral and systemic circulation. Therefore, the potential long-term safety issues of CGRP antagonists could be raised in patients with coronary artery

\section{REFERENCES}

1. Goadsby PJ, Edvinsson L, Ekman R. Vasoactive peptide release in the extracerebral circulation of humans during migraine headache. Ann Neurol. (1990) 28 183-7. doi: 10.1002/ana.410280213

2. Lassen L, Haderslev P, Jacobsen V, Iversen H, Sperling B, Olesen J. CGRP may play a causative role in migraine. Cephalalgia. (2002) 22:54-61. doi: 10.1046/j.1468-2982.2002.00310.x

3. Asghar MS, Hansen AE, Amin FM, van der Geest RJ, Koning P, Larsson HB, et al. Evidence for a vascular factor in migraine. Ann Neurol. (2011) 4:635-45. doi: 10.1002/ana.22292

4. Hansen JM, Hauge AW, Olesen J, Ashina M. Calcitonin gene-related peptide triggers migraine-like attacks in patients with migraine with aura. Cephalalgia. (2010) 30:1179-86. doi: 10.1177/0333102410 368444 and cerebrovascular diseases and could be involved in guideline statements $(23,24)$.

The strength of the present study is the accurate description of the effects of CGRP in healthy human subjects. The potential limitations of the study are the low number of subjects and the lack of a control group.

\section{CONCLUSIONS}

In conclusion, we found out that an intravenous $\alpha$ CGRP infusion might induce vasodilatation of MCA and PCA. It produces systemic effects with a significant decrease of MAP. The Et- $\mathrm{CO}_{2}$ decrease might have a compensatory role in preserving cerebral blood flow and intracranial pressure during CGRP stimulation. The $\alpha$ CGRP could be used as a marker for treatment efficacy of anti-CGRP monoclonal antibodies in migraine patients.

\section{DATA AVAILABILITY STATEMENT}

The raw data supporting the conclusions of this article will be made available by the authors, without undue reservation.

\section{ETHICS STATEMENT}

The studies involving human participants were reviewed and approved by The National Medical Ethics Committee of the Republic of Slovenia. The participants provided their written informed consent to participate in this study.

\section{AUTHOR CONTRIBUTIONS}

BŽ, MZa, and DV contributed to conception and study design. DV organized database and managed experiments. MZa performed the statistical analyses and wrote the results section. DV wrote the first draft of the manuscript. All authors contributed to the article and approved the submitted version.

\section{ACKNOWLEDGMENTS}

The authors express gratitude to Valentin Beznik for technical assistance, and special thanks to all study volunteers.

5. Goadsby PJ, Edvinsson L. The trigeminovascular system and migraine: studies characterizing cerebrovascular changes seen in humans and cats. Ann Neurol. (1993) 33:48-56. doi: 10.1002/ana.410330109

6. Christensen CE, Younis S, Deen M, Khan S, Ghanizada H, Ashina M. Migraine induction with calcitonin gene-related peptide in patients from erenumab trials. J Headache Pain. (2018) 19:105. doi: 10.1186/s10194-018-0927-2

7. Lassen LH, Jacobsen VB, Haderslev, Sperling B, Iversen HK, Olesen J, et al. Involvement of calcitonin gene-related peptide in migraine: regional cerebral blood flow and blood flow velocity in migraine patients. J Headache Pain. (2008) 9:151-7. doi: 10.1007/s10194-008-0036-8

8. Petersen KA, Lassen LH, Birk S, Lesko L, Olesen J. BIBN4096BS antagonizes human $\alpha$-calcitonin gene related peptide-induced headache and extracerebral artery dilatation. Clin Pharmacol Ther. (2005) 77:202-13. doi: 10.1016/j.clpt.2004.10.001 
9. Asghar MS, Hansen AE, Kapijimpanga T, van der Geest RJ, van der Koning P, Larsson HBW, et al. Dilatation by CGRP of middle meningeal artery and reversal by sumatriptan in normal volunteers. Neurology. (2010) 75:1520-6. doi: 10.1212/WNL.0b013e3181f9626a

10. Perko D, Pretnar-Oblak J, Šabovič M, Žvan B, Zaletel M. Differences between cerebrovascular reactivity to L-arginine in the anterior and posterior cerebral circulation. Cerebrovasc Dis. (2011) 31:358-64. doi: 10.1159/000322562

11. Russel FA, King R, Smillie SJ, Kodil X, Brain SD. Calcitonin gene-related peptide: physiology and pathophysiology. Physiol Rev. (2014) 94:1099-142. doi: 10.1152/physrev.00034.2013

12. Edvinsson L, Haanes KA, Warfvinge K, Krause DN. CGRP as the target of new migraine therapies - successful translation from bench to clinic. Nat Rev Neurol. (2018) 14:338-50. doi: 10.1038/s41582-018-0003-1

13. Hoiland RL, Ainslie PN. CrossTalk proposal: the middle cerebral artery diameter does change during alterations in arterial blood gases and blood pressure. J Physiol. (2016) 594:4073-5. doi: 10.1113/JP271981

14. Edvinsson L, Ekman R, Jansen I, McCulloch, Uddman R. Calcitonin generelated peptide and cerebral blood vessels: distribution and vasomotor effects. J Cereb Blood Flow Metab. (1987) 7:720-8. doi: 10.1038/jcbfm.1987.126

15. Erdling A, Sheykhzade M, Edvinsson L. Differential inhibitory response to telcagepant on $\alpha$ CGRP induced vasorelaxation and intracellular $\mathrm{Ca}^{2+}$ level in the perfused and non-perfused isolated rat middle cerebral artery. J Headache Pain. (2017) 18:61. doi: 10.1186/s10194-017-0768-4

16. Ornello R, Frattale I, Caponnetto, V, Pistoia F, Sacco S. Cerebral vascular reactivity and the migraine-stroke relationship: a narrative review. J Neurol Sci. (2020) 414:116887. doi: 10.1016/j.jns.2020.116887

17. Ando K, Pegram BL, Frohlich ED. Hemodynamic effects of calcitonin generelated peptide in spontaneously hypertensive rats. Am J Physiol. (1990) 258:R425-9. doi: 10.1152/ajpregu.1990.258.2.R425

18. Wolf ME. Functional TCD: regulation of cerebral hemodynamiccerebral autoregulation, vasomotor reactivity, and neurovascular coupling. Front Neurol Neurosci. (2015) 36:40-56. doi: 10.1159/0003 66236
19. Rivera-Lara L, Zorrilla-Vaca A, Geocadin R, Ziani W, Healy R, Thompson $\mathrm{R}$, et al. Predictors of outcome with cerebral autoregulation monitoring: a systematic review and meta-analysis. Crit Care Med. (2017) 45:695-704. doi: 10.1097/CCM.0000000000002251

20. Hoiland RL, Fisher JA, Ainslie PN. Regulation of the cerebral circulation by arterial carbon dioxide. Compr Physiol. (2019) 9:1101-54. doi: 10.1002/cphy.c180021

21. Edvinsson L, Fredholm BB, Hamel E, Jansen I, Verrecchia C. Perivascular peptides relax cerebral arteries concomitant with stimulation of cyclic adenosine monophosphate accumulation or release of an endothelium-derived relaxing factor in cat. Neurosci Lett. (1985) 58:213-7. doi: 10.1016/0304-3940(85)90166-1

22. Cottrell GS. CGRP receptor signalling pathways. Handb Exp Pharmacol. (2019) 255:37-64. doi: 10.1007/164_2018_130

23. Tiseo C, Ornello R, Pistoia F, Sacco S. How to integrate monoclonal antibodies targeting the calcitonin gene-related peptide or its receptor in daily clinical practice. J Headache Pain. (2019) 20:49. doi: 10.1186/s10194-019-1000-5

24. Sacco S, Bendtsen L, Ashina M, Reuter U, Terwindt G, Mitsikostas DD, et al. European headache federation guideline on the use of monoclonal antibodies acting on the calcitonin gene related peptide or its receptor for migraine prevention. J Headache Pain. (2019) 20:6. doi: 10.1186/s10194-018-0955-y

Conflict of Interest: The authors declare that the research was conducted in the absence of any commercial or financial relationships that could be construed as a potential conflict of interest.

Copyright (C) 2020 Visočnik, Žvan, Zaletel and Zupan. This is an open-access article distributed under the terms of the Creative Commons Attribution License (CC BY). The use, distribution or reproduction in other forums is permitted, provided the original author(s) and the copyright owner(s) are credited and that the original publication in this journal is cited, in accordance with accepted academic practice. No use, distribution or reproduction is permitted which does not comply with these terms. 